\title{
ENSAYOS
}




\section{LA EDUCACION PRIMARIA Y SECUNDARIA DURANTE EL PRIMER GOBIERNO DE ALFONSO LOPEZ PUMAREJO}

(1934-1938)

(Proyectos y realizaciones)

Aline Helg*

\section{Introducción}

\section{A. Situación de la Educación en 1934}

Cuando Alfonso López Pumarejo tomó posesión de la presidencia de la República en 1934, la situación educativa global de Colombia era la siguiente:

La población mayor de diez años era en un 50\% analfabeta, con un acentuado predominio del analfabetismo en las zonas rurales. De los niños en edad escolar, 513.775 estaban matriculados, mientras que 950.000 carecían totalmente de contacto con el sistema educativo. Si se contara un maestro por sesenta alumnos, faltarían quince mil escuelas y un número igual de maestros para estar en medida de ofrecer un mínimo de educación elemental a la población de siete años de edad ${ }^{1}$. Además, sólo el $7 \%$ de los matriculados estudiaban más allá del tercer año, por falta de oferta educativa en los grados superiores de la primaria. Realmente, de los niños en edad escolar, sólo una cuarta parte asistía a la escuela primaria.

La secundaria oficial contaba con 9.782 alumnos matriculados, a los cuales se sumaban 21.340 inscritos en los colegios privados. De cada 100 alumnos matriculados en la primaria, dos entraban en la secundaria oficial y cuatro en la privada. Fuera del bachillerato clásico, la educación media oficial tenía 500 alumnos en las tres "normales" nacionales, 7.440 matriculados en las "escuelas complementarias", y unos 1.700 alumnos en las "escuelas de artes y oficios"; la enseñanza oficial agropecuaria y comercial era totalmente marginal.

\section{B. El programa de Alfonso López Pumarejo}

Cuando Alfonso López tomó posesión de la presidencia, en agosto de 1934, anunció en su discurso al Congreso que daría prioridad a la educación nacional durante su mandato. En efecto, afirmó que el atraso de Colombia no era debido a otros factores en boga en la época, como la geografía del país, sus climas, la raza colombiana, etc., sino a la falta de preparación de su gente y al desconocimiento del país. Definió así la primera obligación del gobierno: "preparar a los ciudadanos para que sepan aprovechar las riquezas del país y para que sus actividades no sean un penoso arar que no produzca resultados proporcionados al esfuerzo que demandan"2. En otros términos, López se proponía educar a los colombianos para desarrollar el país con una máxima rentabilidad; por eso había que "formar administradores, financistas y diplomáticos, lo mismo que soldados, aviadores y marinos, que artesanos y agricultores, que obreros técnicos y empleados" ${ }^{3}$.

\footnotetext{
${ }^{*}$ Historiadora. Suiza. Candidata a Doctor de la Universidad de Londres.

${ }^{1}$ Educación, Año I, No. 3, Octubre 1933, p. 129 y Ministerio de Educación Nacional, Memoria de 1932, Bogota, 1932, cuadros en anexo.

${ }^{2}$ Alfonso López Pumarejo, "Discurso Pronunciado el 7 de Agosto de 1934 ante el Congreso Nacional", en La Política Oficial. Bogotá, 1935, Vol. 1, p. 30.

${ }^{3}$ Ibid., p. 31. 
A fines del año, el Presidente anunció en el Congreso una campaña de democratización de la enseñanza colombiana y dio más elementos para interpretar la política educativa que se proponía realizar:

"La economía nacional sobrelleva la carga pesada de sostener a esa vasta clase económica miserable que no lee, que no escribe, que no se viste, que no se calza, que apenas come, que permanece involuntaria aunque no muy conscientemente, al margen de la actividad de los dos millones de colombianos que escasamente pueden calcularse en pleno uso de sus atributos ciudadanos. Y las clases explotadoras de la gran industria agrícola ven con satisfacción la abundancia de jornales ínfimos. Sólo cuando estudian inversiones en otro género de industrias comprenden que el país está aniquilando metódicamente al consumidor, y que el círculo vicioso de nuestra organización económica se va cerrando contra el capital casi con la misma rigidez con que se abatió sobre el proletariado" 4 .

El programa aparece entonces más claro: desarrollar y modernizar el país, para volverlo competitivo en el mercado capitalista mundial, sin tocar las bases de su estructura social. En realidad se trata de realizar la integración de Colombia, en lo social, económico y territorial: "elevar el nivel físico, intelectual y económico del pueblo, incorporándolo a la Nación simultáneamente con los extensos territorios que están esperando dueños y cultivadores" ${ }^{5}$, dice Alfonso López. Esa labor de integración nacional justifica la ampliación de las funciones y del intervencionismo del Estado, el aumento de sus gastos; entre éstos, López dará una mayor importancia a los de educación nacional, que él considera como clave en la modernización del país: por la educación se propone integrar a la mayoría de los colombianos que todavía vivían al margen de la sociedad: los campesinos y las mujeres.

Sin embargo, Alfonso López no tiene un plan concreto para la realización de su programa ${ }^{6}$. Sólo se pueden destacar unas líneas generales: una educación más técnica y práctica, tanto para niños como para adultos, un esfuerzo hacia el campesinado y la mujer, la progresión de los gastos públicos en educación.

\section{Prioridades y posibilidades}

\section{A. El sistema educativo}

La decisión más trascendental que tomó el gobierno de Alfonso López para democratizar la educación fue la ley 32 de 1936 sobre igualdad de condiciones para el ingreso a los establecimientos de educación. La ley acabó con la negación de recibir alumnos en las escuelas primarias, secundarias o profesionales "por motivo de nacimiento ilegítimo, diferencias sociales, raciales o religiosas"7. En los establecimientos oficiales, la violación de esta disposición significaba la pérdida del derecho de enseñar en el sector oficial para el maestro responsable; en los planteles particulares, implicaba la supresión de la subvención oficial y del reconocimiento de los títulos expedidos por dicho plantel.

A la par de esa disposición de democratización formal, una apertura real del sistema educativo a los excluidos hubiera requerido otras medidas de la administración López. Se

\footnotetext{
${ }^{4}$ Alfonso López P., "Mensaje presidencial al Congreso sobre Educación Nacional”, en Ibid., p. 140.

${ }_{6}^{5}$ Ibid., p. 142.

${ }^{6}$ Ibid., p. 143

${ }^{7}$ Ley 32 de 1936 en MEN, Educación colombiana, 1903-1958, Bogotá, 1959, TI., p. 129. 
trataba sobre todo de empezar a abrir la instrucción primaria al millón de niños que permanecían eliminados de ella, creando escuelas y formando maestros. Era urgente crear un servicio médico y restaurantes escolares, dadas las condiciones de salud deficientes de una proporción importante de los alumnos. Se trataba también de ampliar las carreras intermedias en industrias, agricultura, comercio, y mejorar el sistema de las escuelas complementarias.

Sin embargo, la. Constitución de 1886, el Concordato con la Santa Sede de 1887 y la ley 39 de 1903 que organizaba la educación, vigentes en 1934, limitaban considerablemente la facultad de intervención de la Nación en materia educativa. En primer lugar, la educación pública debía estar en concordancia con la religión católica; ello significaba que cualquier reforma educativa debía ser aprobada por la Curia. En efecto, el arzobispo de Bogotá tenía derecho de control sobre el contenido moral y religioso de todos los niveles de enseñanza; además, la "concordancia con la religión católica" suscitaba diversas interpretaciones en cuanto a la educación privada y al papel docente del Estado.

En segundo lugar, la descentralización de la educación oficial y la multitud de fuentes de poder y de financiamiento en la materia impedían al Estado una política activa. En la instrucción primaria, la Nación establecía los programas y financiaba los útiles y textos; el Departamento ejercía la inspección escolar, nombraba a los maestros y pagaba sus salarios; el municipio estaba encargado de suministrar los locales y muebles escolares. La secundaria nacional era costeada y vigilada por la Nación, mientras que la normalista nacional era costeada por la Nación pero inspeccionada por el Departamento; sin embargo, en ambos casos, silos planteles eran departamentales o municipales, no dependían del Ministerio de Educación. Finalmente la educación industrial, comercial, agrícola y universitaria era costeada con fondos nacionales si los establecimientos funcionaban en Bogotá, con fondos departamentales o municipales en los demás casos ${ }^{8}$.

En concreto, la legislación significaba que las partidas nacionales para la educación eran inferiores a las departamentales que estaban casi totalmente destinadas a costear los salarios de los maestros; los municipios consagraban generalmente una parte mínima de sus presupuestos a los locales escolares. Por consiguiente, las desigualdades regionales se reflejaban en la oferta escolar. Con un sistema educativo multicéfalo, el poder real del Ministerio de Educación era limitado. Aunque la reforma de 1932 había establecido una inspección nacional a todos los niveles de educación, por razones de presupuesto, sólo se creó la inspección nacional prevista para la educación primaria y normalista.

Sin embargo, un medio de desarrollar una política educativa firme era nombrar un ministro de educación especialista en la materia, poco político, que permaneciera en el cargo durante el cuatrienio de la presidencia de López. Era la única manera de mantener una orientación definida a las reformas, pues los funcionarios del Ministerio, a causa del clientelismo político, cambiaban con mucha frecuencia. Alfonso López, no obstante, nombró ocho ministros durante su primer gobierno, de los cuales sólo Darío Echandía permaneció más de un año (ver cuadro No. 1).

\footnotetext{
${ }^{8}$ Ley 39 de 1903 en Ibid., pp. 9-14. 


\section{CUADRO No. 1 \\ MINISTROS DE EDUCACION NACIONAL, 1934.1938*, CON FECHA DE NOMBRAMIENTO}

Carlos Lozano y Lozano
Luis López de Mesa
Darío Echandía
Alberto Lleras Camargo
Tulio Enrique Tascón
Jorge Zalamea
Alejandro López
José Joaquín Castro
Martínez

Carlos Lozano y Lozano

$\begin{array}{ll}7 \text { agosto } & 1934 \\ 13 \text { agosto } & 1934 \\ \text { 10agosto } & 1935 \\ 28 \text { enero } & 1937 \\ 27 \text { febrero } & 1937 \\ 1 \text { marzo } & 1937 \\ 27 \text { marzo } & 1937 \\ 4 \text { junio } & 1937\end{array}$

* Todos de filiación liberal

Fuente: $\quad$ Datos básicos para la elaboración de los índices de Política de Compromiso, Bogotá, Universidad de los Andes, Departamento de Ciencia Política, mimeógrafo no publicado.

La segunda condición para implementar un programa educativo era dotar el Ministerio de Educación con medios financieros suficientes. Las cifras del presupuesto nacional de educación y su proporción entre los gastos totales de la Nación muestran la voluntad gubernamental en ese campo:

\section{CUADRO No. 2}

\section{GASTOS NACIONALES GENERALES Y EN EDUCACION, 1934-1939}

Gastos Generales
(en pesos)

(1)

$\begin{array}{lc}1934 & 74.322 .712 \\ 1935 & 60.313 .057 \\ 1936 & 72.488 .425 \\ 1937 & 83.947 .430 \\ 1938 & 92.618 .845 \\ 1939 & 102.057 .814\end{array}$

\section{Gastos para \\ Educación \\ (en pesos)}

(2)

1.543.019

2.531 .724

5.310 .754

6.182 .902

7.608.862

8.787 .901

\section{\% Educación en Gastos Generales}

(2)

(1)

2.08

4.20

7.33

7.37

8.64

8.86

Fuente: Luis A. Bermúdez, "Diez Años de Educación y Cultura en Colombia”, en Anales de Economía y Estadística, TV., Nos. 3-4, 1941, p.

El progreso, tanto en las sumas dedicadas a la educación como en relación a los gastos totales de la Nación, es evidente y constante. Eso fue consecuencia no solamente de la conciencia creciente entre los dirigentes de la necesidad de fomentar la enseñanza pública, sino también de la reforma tributaria que creó nuevas rentas para el Estado (por ejemplo el impuesto de rentas y sucesiones). Sin embargo, sólo en 1962 se dio cumplimiento a la Ley 12 de 1939, la cual estipulaba que del año 1936 en adelante, la Nación invertiría no menos del 100/o de su presupuesto general en la educación pública. 
A nivel departamental, el esfuerzo fue notable, aunque en proporciones menores:

\section{CUADRO No. 3 \\ GASTOS DEPARTAMENTALES PARA EDUCACION GENERAL Y PRIMARIA, 1934-1938}

Año $\quad \begin{gathered}\text { Gastos Departamentales } \\ \text { para Educación General } \\ \text { (en pesos) }\end{gathered}$

1934

1935

1936

1937

1938

6.401 .394
7.191 .125
7.514 .658
8.868 .073
9.300 .982

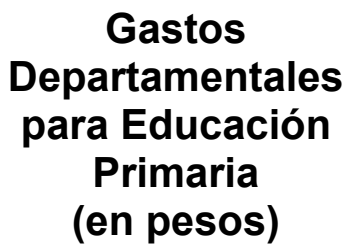

4.884 .341

5.411266

6.386 .678

6.936 .579

Fuente: M.E.N., Memoria de 1938, Bogotá, 1938, pp. 12-13 y Memoria de 1942, Bogotá, 1942, p. 5. También Yvón Lebot, Elementos para la Historia de la Educación en Colombia en el Siglo XX, Bogotá, DANE, S.F., p. 131.

A pesar del esfuerzo por aumentar los presupuestos educativos, estas sumas eran muy inferiores ante las necesidades de la instrucción pública.

\section{B. Alfabetización y cultura popular}

La lucha del gobierno de López contra el analfabetismo fue casi nula. Su única manifestación fue la creación por decreto del servicio escolar voluntario ${ }^{9}$. La idea era establecer un servicio social femenino y vincular las mujeres a la comunidad social y al proceso de desarrollo por medio de la alfabetización de los niños entre siete y veinte años que no podían asistir a la escuela. Las alfabetizadoras tenían que ser colombianas, de dieciocho a cuarenta años y sanas; debían haber cursado cuatro años de instrucción primaria y no ser maestras. Las mujeres recibirían de la Nación un peso por niño alfabetizado. Todo el servicio voluntario escolar estaba colocado bajo el control de las direcciones departamentales de educación. Sin embargo, el decreto permaneció "letra muerta": no existió una sola mujer alfabetizadota en todo el territorio nacional.

Es notable que una de las mayores realizaciones de la Revolución en Marcha en favor de la educación popular - la Comisión de Cultura Aldeana- no incluyera en sus funciones un programa de alfabetización. Cuando Luis López de Mesa creó esa institución, tenía ala vista un plan global de integración de la aldea colombiana. Su fin era desarrollar la vida social local por medio de una Casa Social, una banda de música, la radio y el cinematógrafo, una biblioteca, un médico oficial, un abogado de los pobres, etc.

La escuela tenía una función central: vincular la labor del maestro a las del párroco, del médico y del alcalde. La Comisión Nacional de Cultura Aldeana estaba compuesta de cinco expertos: un urbanista, un salubrista, un agrónomo, un pedagogo y un sociólogo. Adelantó las primeras investigaciones sociológicas concretas sobre educación en Colombia, a partir de un estudio a nivel departamental con entrevistas y observaciones

\footnotetext{
${ }^{9}$ Decreto No. 1458 de 1936, en Ibid, pp. 138-139,
} 
directas. Las conclusiones de la Comisión debían servir la orientación de la política del gobierno. Pero solamente salieron dos estudios: uno sobre Huila, otro sobre Nariño ${ }^{10}$.

\section{Educación primaria}

\section{A. Condiciones Generales}

A nivel de la educación primaria, como ya lo hemos visto, el margen de acción del Estado se limitaba al suministro de material de enseñanza, al establecimiento de programas escolares y a la inspección escolar. El gobierno de Alfonso López buscó, pues, una vía para realizar su programa educativo dentro de ese marco.

Una primera medida fue la de cumplir con el artículo 10o. de la ley 39 de 1903, el cual imponía a la Nación la provisión de los textos y útiles de enseñanza. En efecto, las partidas antes apropiadas para ese renglón eran tan reducidas que no alcanzaban para un cuaderno por alumno matriculado (ver cuadro No. 4).

Entre 1934 y 1935, la partida para estos gastos se multiplicó por más de 10, y representó entonces casi un peso por alumno, lo que era considerado como el mínimo necesario para equipar correctamente los alumnos en cuadernos, lápices, tizas, etc. Sin embargo es de anotar que los gastos en útiles nunca volvieron a conocer su nivel de 1935 antes de 1951.

\section{CUADRO No.4 \\ GASTOS DE LA NACION EN UTILES Y TEXTOS DE ENSEÑANZA 1934-1939}

$\begin{array}{rr}\text { Año } & \text { Gastos (en pesos) } \\ 1934 & 43.795 \\ 1935 & 465.279 \\ 1936 & 361.064 \\ 1937 & 386116 \\ 1938 & 246.557 \\ 1939 & 405.000\end{array}$

Fuente: $\quad$ Ministerio de Educación Nacional, Memoria de 1938-1939, Bogotá, 1939, p. 23.

Paralelamente, el Ministerio de Educación trató de organizar la distribución del material que era totalmente anárquica. Un jefe de los depósitos del Ministerio estuvo encargado de repartir los útiles entre los departamentos en función de su población escolar; a nivel departamental, el director de educación y un empleado encargado del recibo y de la distribución del material debían repartirlo a las escuelas según el número de matrículas; finalmente, los maestros tenían que dar cuenta del material recibido a las direcciones departamentales de educación y éstas transmitirlas al Ministerio ${ }^{11}$. Por otra parte, el Ministerio inició la publicación de la revista infantil Rin-Rin, medida de emergencia destinada a solucionar provisionalmente el problema de la falta de buenos textos educativos nacionales. La revista, publicación lujosa y a veces de difícil acceso por su lenguaje, fue bastante criticada; apareció regularmente en 1936, para declinar después y desaparecer definitivamente en 1938.

\footnotetext{
${ }^{10}$ Ley 12 de 1934, en Ministerio de Educación Nacional, Memoria de 1935, Bogotá, 1935, pp. 59-64.

${ }^{11}$ Decreto No. 1329 de 1936 en Educación Colombiana, op. cit., pp. 137-8. 
Cuando Alfonso López Pumarejo inició su gobierno, la inspección escolar estaba en decadencia por razones principalmente fiscales. No obstante la reforma de 1932 y la presencia del educador Agustín Nieto Caballero en la cabeza de ese departamento, el control del Estado sobre la educación pública era casi inexistente, y muchas veces, cuando existió, fue un instrumento de juegos políticos. En 1935, el liberal Darío Echandía, desde el Ministerio, intentó centralizar la educación, pero se enfrentó a la oposición de los departamentos, sobre todo de los ricos y conservadores como Antioquia, que temían retroceder en su desarrollo educativo y perder su independencia ${ }^{12}$. Finalmente el Ministro no tuvo otro medio que limitarse a reglamentar las funciones de la inspección nacional ya definidas por la ley orgánica de 1903, extendiéndolas a los métodos de enseñanza y a la asistencia social escolar ${ }^{13}$. Este decreto permitió el nombramiento de los inspectores nacionales, el cual no se hizo siempre según criterios de eficiencia. En efecto, los conflictos en el interior del partido de gobierno y el habitual juego clientelista llevaron el Ministerio a prescindir de la colaboración de Agustín Nieto Caballero, quien se había distinguido en su cargo de inspector nacional por un conocimiento directo de las necesidades educativas en casi todas las regiones del país.

Que la inspección nacional se volvió más eficaz después de esta reforma parece dudoso. Ella siguió a la vez atribución de la Nación y de los departamentos. A nivel departamental, la inspección variaba mucho según el presupuesto, la política y el grado de interés por la educación de la gobernación de cada departamento. Además la dualidad de la inspección real de las escuelas, función de la Nación y del departamento, llevó a no pocos conflictos entre los inspectores de la una y del otro. En Cundinamarca, por ejemplo, cada inspector debía teóricamente visitar nueve municipios y un promedio de 95 escuelas al año... En realidad, las escuelas próximas a la capital recibían varias visitas anuales, mientras que los maestros de las otras llenaban un informe de visita que les había enviado el inspector encargado ${ }^{14}$.

Quedaba al gobierno de López el arma de la orientación de los programas de educación. Pero las condiciones del país la condenaban a ser un arma muy débil y teórica. En efecto, ¿cómo lograr cambios de pénsum con un magisterio sin formación pedagógica en su $73 \%$, además instalado en una rutina difícil de modificar; con un sistema educacional desprovisto de un control nacional que lo articulara; y sobre todo con una red de comunicaciones tan precaria que, salvo en los centros urbanos importantes ${ }^{15}$, mantenía a los maestros aislados? Así, las reformas de programa no llegaban sino a las zonas urbanas de los departamentos más desarrollados.

El ejemplo más ilustrativo de la dimensión del problema fue el intento de introducir los métodos de la "escuela nueva" en la primaria y los "centros de interés" del belga Decroly. El Gimnasio Moderno, colegio privado de vanguardia fundado en Bogotá por Agustín Nieto Caballero en 1914, había sido el primero en aplicar el método, particularmente después de la visita de Ovidio Decroly al Gimnasio en 1925. La pedagogía decroliana considera el desarrollo educativo en tres etapas: la observación, la asociación y la expresión; se enseña primero a los niños a observar la realidad, después a asociar esa observación a la teoría y finalmente se lleva a la expresión los hechos adquiridos mediante la observación y la asociación. Para realizar su teoría, Decroly creó los "centros de interés", que educan al niño alrededor de las actividades básicas de la vida como

\footnotetext{
${ }^{12}$ El Tiempo, 27, Xl. 35.

${ }^{13}$ Decreto No. 620 de 1936, en Educación Colombiana, op. cit., pp. 135-136.

${ }^{14}$ El Tiempo, 2. V. 38.

${ }^{15}$ En 1938, 16 ciudades tenían más de 20.000 habitantes. 
alimentarse, vestirse, protegerse de los peligros, trabajar. De ahí, y gracias al método de globalización, se extiende el interés de los alumnos a materias típicamente escolares ${ }^{16}$.

Desde 1932 un plan de estudios basado en la "escuela nueva", era ensayado en algunas escuelas oficiales bajo la supervisión del Ministro de Educación Carrizosa Valenzuela y del educador Agustín Nieto Caballero. En 1935, el Ministerio de Educación quiso declarar terminado el período de ensayo y adoptar el programa a nivel nacional. Empero no se tomo mayor decisión que recomendar el método a los departamentos. Ciertas regiones mas avanzadas en materia educativa, tales como Antioquia, Boyacá, Caldas y el Valle, introdujeron los "centros de interés" en algunas de sus escuelas urbanas. Sin embargo, dada la proximidad de Bogotá, el único departamento que oficializó el método fue Cundinamarca a partir de 1936-1937; ello es muy significativo del verdadero radio de acción del Ministerio de Educación. A través de boletines, la Secretaría de Educación de Cundinamarca intentó explicar e implantar la nueva pedagogía. Pero su método y contenido desorientaron a un Magisterio poco o nada preparado para entender y menos aplicar la teoría al medio ambiente. En realidad, la pedagogía decroliana solamente tuvo éxito en escuelas y colegios con personal docente capacitado. La causa del fracaso a nivel oficial residió principalmente en la falta de orientación de los maestros: habría sido preciso empezar la reforma en los institutos normales, formando un personal docente especializado, y no directamente en las escuelas. Pero a la vez ese fracaso, restringido a la poca cobertura de la reforma Cundinamarca- muestra los estrechos límites del poder del Ministerio de Educación.

Otro de los problemas permanentes de la educación primaria era el de las instalaciones. En 1936, en su Memoria de Educación, Darío Echandía denunciaba la falta de 15.000 locales para el millón de niños que no concurrían a la escuela y el hecho que el $900 \%$ de las 8.000 escuelas existentes funcionaban en locales inadecuados. Una campaña de reparación y construcción escolar era pues urgente.

La causa principal de este problema radicaba en que la fuente exclusiva de financiamiento de las obras era el municipio. Según la ley, éstos debían dedicar el 50 /o de lo recaudado por impuesto predial a las instalaciones escolares ${ }^{17}$. Muchos municipios no lo cobraban por falta de catastro, otros lo cobraban irregularmente. En general la situación reflejaba la desigualdad entre las distintas regiones del país. Pero la pobreza de numerosos municipios no explica todo; había entre ellos un gran desinterés por la educación que se manifestaba en la costumbre de manipular los fondos educativos. Preferían gastar en obras de prestigio o en fiestas y conmemoraciones útiles a la consecución de votos para los concejales.

Hasta 1936, la única manera para la Nación de participar en los gastos de construcción escolar era a través de los auxilios particulares votados por el Congreso. En consecuencia, una intervención estatal más institucionalizada era necesaria. El Ministerio de Educación decidió atribuir una partida fija de su presupuesto para auxiliar a la construcción de escuelas. En contrapartida, el Ministerio se reservaba la facultad de vigilar los planos y la ejecución de las edificaciones que contribuía a financiar ${ }^{18}$. En 1937 la contribución nacional representaba unos 200.000 pesos y en 1938, 300 mil $^{19}$. En realidad, esa legislación fue el primer paso hacia una financiación y vigilancia estatal de la construcción escolar que se realizara durante el gobierno de Eduardo Santos con el

\footnotetext{
${ }^{16}$ Cf. Ministerio de Educación Nacional, El Doctor Decroly en Colombia, Bogotá, 1932

${ }^{17}$ Ordenanza 17 de 1915 en M.E.N., Memoria de 1918, Bogotá, 1918, p. 60.

${ }^{18}$ Decreto No. 1122 de 1936, en Educación Colombiana, op. cit., pp. 136-7.

${ }^{19}$ M.E.N., Memoria de 1938-1939, Bogotá, 1939, p. 9.

Digitalizado por RED ACADEMICA
} 
decreto No. 503 sobre Fondo de Fomento Municipal de 1940 y se complementara en 1944 por la ley 30.

No parece que con la aplicación, desde 1937, del decreto No. 1.122 se haya registrado un incremento en la construcción escolar mayor del que se venía dando hasta ese año (ver cuadro No. 5). Sin embargo, es de suponer que la vigilancia nacional impuesta por el mismo decreto haya redundado más bien en un incremento de la calidad de las obras.

\section{CUADRO No.5 \\ NUMERO DE LOCALES ESCOLARES CONSTRUIDOS O EN CONSTRUCCION EN LOS DEPARTAMENTOS, 1934-1938 (HASTA MAYO)}

$\begin{array}{lccccc}\text { Años } & 1934 & 1935 & 1936 & 1937 & 1938 \text { (hasta mayo } \\ \text { Numero de locales } & 117 & 116 & 137 & 149 & 86\end{array}$

Fuente: $\quad$ M.E.N., Memoria de 1938, Bogotá, 1938, cuadro en anexo.

Sí se considera el número global de escuelas primarias oficiales entre esos años, los logros en oferta escolar aparecen concentrados en las zonas rurales (ver cuadro No. 6).

En 1938 las escuelas urbanas no superan el nivel de 1934, mientras que los planteles rurales sí aumentan en la proporción del 17.60/o en cuatro años. Ese aumento fue superior al de los años 1918-1934 en los cuales el aumento promedio anual fue de 161 escuelas $^{20}$ entre 1934 y 1938 este promedio alcanza las 218 escuelas anuales.

\section{CUADRO No.6 \\ NUMERO DE ESCUELAS PRIMARIAS OFICIALES, 1934-1938}

$\begin{array}{rrrc}\text { Año } & \begin{array}{c}\text { Escuelas } \\ \text { Urbanas }\end{array} & \begin{array}{c}\text { Escuelas } \\ \text { Rurales }\end{array} & \begin{array}{c}\text { Total } \\ \text { Escuelas }\end{array} \\ 1934 & 2664 & 4858 & 7522 \\ 1935 & 2773 & 5146 & 7919 \\ 1936 & 2749 & 5565 & 8314 \\ 1937 & 2619 & 5396 & 8015 \\ 1938 & 2664 & 5714 & 8378\end{array}$

Fuente: $\quad$ Luis A. Bermúdez, op. cit., p. 52.

Entre 1934 y 1938 la matrícula global en la primaria oficial aumentó del 9.9\%, pero como en el caso del número de escuelas, el fenómeno se manifestó sobre todo en el campo, donde la matrícula creció del $18 \%$, mientras que en las zonas urbanas solo aumentó del $1 \%$; el progreso de a matrícula femenina es un poco más alto que el de la masculina (11.3\% contra $8.7 \%$ ), concentrado en las escuelas rurales donde las niñas logran representar el $49 \%$ del alumnado en 1938. Es evidente que el esfuerzo se concentró hacia las zonas rurales. Sin embargo, a pesar de que teóricamente el decreto No. 1487 de 1932 había unificado la primaria urbana y rural con cuatro años de enseñanza, la desigualdad entre el campo y la ciudad permanecía; indicativo de ello es el hecho de que

\footnotetext{
${ }^{20}$ En 1918 había 4.940 escuelas primarias oficiales; en 1934, 7.522.
} 
el $80 \%$ de las escuelas rurales eran unitarias o alternadas, ofreciendo en realidad un solo año de primaria a los alumnos.

CUADRO No.7

ALUMNOS MATRICULADOS EN LAS ESCUELAS PRIMARIAS OFICIALES, SEGUN SEXO Y ZONA, 1934-1938

\begin{tabular}{|c|c|c|c|c|c|c|c|c|c|}
\hline \multicolumn{5}{|c|}{ Urbana } & & \multicolumn{2}{|l|}{ Total } \\
\hline & lombres & Mujeres & Total & Hombres & Mujeres & Total & Hombres & eres & Total \\
\hline & 132.117 & 120.205 & 252 & 120 & 128.600 & 268.553 & 272.070 & 248.805 & 520.875 \\
\hline & 121 & $118 . \varepsilon$ & 24 & & & & & & 163 \\
\hline 93 & 124.777 & 121.877 & 246.654 & 15 & 14 & 305. & $2 \varepsilon$ & 26 & 551.961 \\
\hline & & & & & & & & & 3.605 \\
\hline $93 \varepsilon_{-2}$ & 130.051 & 125.249 & 255.300 & 165.579 & 151.678 & 317.257 & 295.630 & 276.927 & 572.557 \\
\hline
\end{tabular}

Fuente: Luis A. Bermúdez, op. cit., p. 57.

Prescindiéndose de la cuestión de la calidad de la educación, es preciso anotar que el aumento global de la matrícula en los años de la Revolución en Marcha fue inferior al promedio anual de 3.75\% realizado entre 1918 y 1934. En 1942 el Ministro de Educación, Germán Arciniegas, señaló que el avance de 10\% en la matrícula entre 1935 y 1938 resultaba poco relevante si se lo comparaba con el aumento del $42 \%$ de los presupuestos departamentales para educación primaria ${ }^{21}$.

\section{B. La enseñanza complementaria}

Desde 1932, la enseñanza primaria comprendía tres años, seguidos por dos años de enseñanza complementaria para los alumnos que no hacían estudios secundarios. Las escuelas complementarias en principio debían orientarse hacia las artes y oficios ${ }^{22}$. Organizándose progresivamente, la educación complementaria conoció su nivel más alto en 1935, año a partir del cual estuvo perdiendo importancia. Pero en la década del cuarenta, el Ministerio le dio un nuevo impulso, con la introducción de las escuelas vocacionales agrícolas en 1941.

CUADRO No. 8

\section{ESCUELAS COMPLEMENTAR IAS OFICIALES, 1934-1938}

ESCUELAS

$\begin{array}{llrr}\text { Año } & \text { Tot. } & \text { H } & \text { M } \\ \mathbf{1 9 3 4} & 146 & 77 & 69 \\ \mathbf{1 9 3 5} & 220 & 127 & 93 \\ \mathbf{1 9 3 6} & 188 & 98 & 90 \\ \mathbf{1 9 3 7} & 127 & 65 & 61 \\ \mathbf{1 9 3 8} & 113 & 55 & 55\end{array}$

ALUMNOS

\begin{tabular}{ll} 
Mixt. & Tot. \\
$-\quad 7440$ \\
$-\quad 9014$ \\
$-\quad 8453$ \\
\hline 1 & 5803 \\
3 & 5797
\end{tabular}

$\mathrm{H}$

3969

4847

4369

2738

2433

M
3471
4167
4084
3065
3364

Fuente: Luis A. Bermúdez, op. Cit, p. 63

Entre 1934 y 1938, la matrícula en las escuelas complementarias disminuyó del 22\%, sobre todo la masculina que se redujo en un $39 \%$. Esa disminución se explica en el hecho de que la enseñanza complementaria de artes y oficios (o industrial) resultaba muy costosa por la inversión requerida en maquinaria y material de trabajo, que no estaba al alcance de la Nación entonces. La falta de dotación y de profesorado idóneo y las de-

\footnotetext{
${ }^{21}$ MEN., Memoria de 1942, Bogotá, 1942, p. 5.

22 Decreto 1487 de 1932 en Educación colombiana, op. cit., pp. 97-98 
ficiencias en la organización de los planteles hicieron que la instrucción complementaria en general no se distinguiera de la primaria; además provocaron un deterioro, que solo se recuperará con una orientación más agrícola en la década siguiente.

\section{Higiene y alimentación escolar}

La encuesta que se realizó en Boyacá a fines de los años veinte, base de la reforma educativa de vanguardia que conoció entonces este Departamento, los informes del Ministro de Educación, Carrizosa Valenzuela, y Agustín Nieto Caballero al regresar de su gira en el país, las investigaciones adelantadas por la Comisión de Cultura Aldeana, los artículos de prensa, todos convergían hacia una misma conclusión. Las condiciones de salubridad, higiene y alimentación del pueblo colombiano, principalmente en el campo, eran tan deficientes que una reforma educativa resultaba yana sin un programa de higiene y nutrición paralelo.

En 1934, los únicos departamentos que tenían un servicio médico escolar funcionando eran Boyacá y Cundinamarca; en las otras regiones había solo un médico para todo el departamento, o ninguno. Al mismo tiempo, se evaluaba que un $20 \%$ de los alumnos de las escuelas estaban enfermos; este porcentaje en ciertas partes alcanzaba el $90 \%{ }^{23}$. Una partida de 300.000 pesos fue decidida en 1935 para crear cuarenta comisiones nacionales, compuestas de un médico, un dentista y un inspector escolar, que debían recorrer el país por zonas definidas. También se apropiaron 150.000 pesos para la compra y distribución de drogas para tratamiento de los niños sin recursos económicos ${ }^{24}$. El año siguiente, sin embargo, no se ejecutó la creación de las cuarenta comisiones, por falta de apropiación suficiente; pero se nombraron 17 médicos y el mismo número de dentistas y se proyectó atribuir 120.000 pesos para drogas ${ }^{25}$. Paralelamente se inició una campaña de salubridad con medicina escolar y asistencia campesina. Pero esta campaña, adelantada conjuntamente por la Nación, los departamentos, los municipios e instituciones privadas o semi-oficiales (la Federación Nacional de Cafeteros), careció de coordinación y se perdió en las distintas burocracias ${ }^{26}$.

En 1938, el servicio médico escolar continuaba fundamentalmente bajo la responsabilidad departamental. En consecuencia, Bolívar, Cauca, Nariño, Santander, Tolima y el Valle no tenían servicio médico organizado, mientras que Antioquia, Boyacá, Cundinamarca estaban bien provistos en esto. Sin embargo, globalmente la medicina escolar había conocido en cuatro años un progreso enorme: en 1938 se contaban 86 médicos escolares, 59 dentistas, 56 enfermeras. Además se habían creado tres colonias de vacaciones para niños de precarias condiciones de salud.

Como la higiene, los primeros restaurantes escolares fueron fundados en Boyacá en 1925; más tarde funcionaron en otros departamentos. Pero a nivel nacional fue solo en 1936 que se organizó este servicio. Se estimaba en la época que un alumno sobre cinco iba a la escuela sin haber desayunado; por otra parte, por las distancias en el campo se presentaba el problema del almuerzo de los niños que hacían jornada continua ${ }^{27}$.

El Ministerio de Educación quiso aliviar esta situación, sin relevar la responsabilidad de los padres de familia, de los municipios y de los departamentos. El Estado se limitó a

\footnotetext{
${ }^{23}$ MEN., Memoria de 1935, op. cit., p. 10

${ }^{24}$ Ibid.

${ }^{25}$ La manera de financiarlas era tan vaga y tan a largo plazo (compra de máquinas para la fábrica de calzado en las cárceles y venta de los calzados producidos a las clases bajas) que su realización fue casi nula.

${ }^{26}$ MEN.,Memoriade 1936, Bogotá, 1936, pp. 17-20.

${ }^{27}$ MEN., Memoria de 1935, op. cit., pp. 7-10.

Digitalizado por RED ACADEMICA
} 
auxiliar las iniciativas locales, pero sin convertirse en "una gigantesca mansión de beneficencia" ${ }^{28}$. El decreto No. 219 de 1936 fijó las condiciones para recibir el auxilio de la Nación: tener una granja escolar cuya producción ayudara al funcionamiento del restaurante; contar con auxilios del departamento y del municipio cuyo monto individual fuera por lo menos igual al de la Nación; crear una junta administradora del restaurante responsable frente a las autoridades ${ }^{29}$. De este modo en 1937 funcionaban 638 restaurantes escolares. Estaban sin embargo concentrados en los departamentos más organizados: Antioquia, Cundinamarca y Boyacá los cuales totalizaban 355 de ellos. También funcionaban en Boyacá 299 cooperativas de nutrición (conjuntos de granjas y restaurantes en parte autofinanciados). La mayoría de los restaurantes estaban localizados en las ciudades y las cabeceras municipales, privando en gran parte al campo de estas facilidades. La Nación dio en 1937, 400.000 pesos para la alimentación escolar; los departamentos y los municipios apropiaron 1.100 .000 pesos $^{30}$.

El esfuerzo del gobierno de López para montar y fomentar los restaurantes escolares fue notable. Sin-embargo, al igual que para la higiene, el sistema organizado y el modo de financiamiento utilizado acentuaron las diferencias regionales favoreciendo las zonas urbanas y los departamentos más ricos con mejor dirección educativa. Posteriormente se trató de corregir esa tendencia, atribuyendo los auxilios a los departamentos proporcionalmente a su población escolar ${ }^{31}$. Las partidas nacionales estuvieron declinando poco a poco y no volvieron a conocer su nivel de 1937-1938 antes de 1951.

En resumen, la educación primaría durante la Revolución en Marcha conoció un progreso más cualitativo que cuantitativo: el número de escuelas, maestros y alumnos no creció mucho más que en el período conservador. Sin embargo, la provisión y la distribución de material de enseñanza se mejoró, la construcción escolar empezó a organizarse, se intentó introducir métodos más modernos de enseñanza y se inició un servicio de médicos y restaurantes escolares a nivel nacional.

Es preciso destacar, por otra parte, el esfuerzo del Ministerio de Educación por la enseñanza rural, a costa de la urbana. Esa política nos parece responder a dos orientaciones de la Revolución en Marcha. La primera era la modernización del país, para la cual una mas estrecha vinculación del campo a la economía nacional se hacía necesaria; y esa vinculación exigía la socialización de la población rural por medio de la escuela ${ }^{32}$. La otra era más política: el liberalismo esperaba, mediante la educación rural y otras medidas que tomó en favor del campo (democratización del sufragio masculino, Ley 200 de 1936 por ejemplo), ampliar su base electoral, tradicionalmente más urbana.

\footnotetext{
${ }^{28}$ MEN., Memoria de 1936, op. cit., p. 23.

${ }^{29}$ Decreto No. 219 de 1936 en Educación Colombiana, op. cit., $p .134$

${ }^{30}$ MEN., Memoria de 1938, Bogotá, 1938, p. 37-38 y cuadro en anexo.

${ }^{31}$ Decreto No. 15 de 1939 en Educación Colombiana, op. cit., pp. 228-229.

${ }^{32}$ Véase Alfonso López P., "La Educación Pública Primaria", en Educación, Año III, Nos. 24-25, Julio-Agosto, 1935, pp. $416-417$. 


\section{El magisterio}

\section{A. Los Maestros}

En 1935, la enseñanza primaria oficial contaba 10.604 maestros. Globalmente el magisterio era en sus $30 \%$ graduado, pero la desigualdad entre el campo y las zonas urbanas era fuerte: casi la mitad de los maestros urbanos era normalistas graduados, mientras que solo el $6 \%$ del personal docente rural tenía grado. Es decir que prácticamente la totalidad del magisterio en el campo no tenía ninguna formación pedagógica. Además, de diez maestros, siete eran mujeres solteras; en el campo, el $90 \%$ de los institutores eran de sexo femenino, en las ciudades solamente el $55 \%{ }^{33}$. La no calificación del magisterio colombiano y el peso de trabajo femenino tenían su origen en el nivel de sueldos y el estatuto social de la profesión, tradicionalmente muy bajos en el país, que generaban un círculo vicioso de condiciones socio-económicas inferiores y subcalificación. Así el carácter femenino de la profesión influía también en la consideración social que hombres y sociedad en general tenían de la tarea de educador.

Los sueldos eran costeados por los departamentos. Cada sección del territorio tenía su propio sistema y pagaba un sueldo mínimo al alcance de sus recursos: Nariño 18 pesos mensuales, Antioquia y Caldas 60 pesos. El sueldo de un maestro rural era apenas la mitad del recibido por un maestro en la ciudad. No existía un escalafón nacional de salarios relacionado con la formación y los años de servicio. Además el cumplimiento en el pago de los sueldos dependía del estado fiscal de los departamentos. Por ejemplo, el endeudamiento del fisco departamental influían su capacidad de pago. Muchas veces, las gobernaciones no alcanzaban a realizar los ingresos por impuesto a la producción de licores (fuente principal de financiación del renglón educativo) y tenían que recurrir al pago de sueldo en especies (en este caso, los aguardientes y rones de la región).

La Ley 2a. de 1937 fijó un salario mínimo para los maestros de primaria equivalente a 40 pesos mensuales. Creó el escalafón nacional del magisterio y estableció que podrían ser nombrados maestros de escuela solamente los que llenaban los requisitos fijados en éste. Para su clasificación, impuso un examen de conocimientos a todo el magisterio en ejercicio que provocó una fuerte reacción de los maestros, especialmente los recién salidos de los institutos normales ${ }^{34}$. La aplicación de esta ley fue lenta, dado que el gobierno no tenía los medios para hacerla cumplir. Pero entre 1940 y 1942 ya se habían clasificado los maestros en el escalafón y ya recibían el salario mínimo con mayor puntualidad y en efectivo.

\section{B. La formación de los Maestros}

La solución del problema docente estaba, sin embargo, en la formación de un nuevo magisterio que iría, poco a poco, cerrando la brecha de los 15.000 maestros faltantes. La polémica en la época oponía los defensores de la creación rápida de un magisterio de emergencia y los partidarios de la primacía absoluta de la calidad sobre la cantidad. La legislación promulgada reflejaba esa dualidad de medidas de emergencia y a largo plazo. El decreto No. 1487 de 1932 había reformado la enseñanza normalista: de seis años especializados, la había extendido a seis años de bachillerato y dos años de especialización pedagógica en las nuevas facultades de educación; había creado las carreras de profesor de secundaria y de normal — que no existían antes - con cuatro años de especialización en las Facultades. También había organizado cursos de

\footnotetext{
${ }^{33}$ MEN., Memoria de 1935, op. cit., pp. 250-251.

${ }^{34}$ Véase la interesante Biografía de Ester Gónima, Una Maestra, Una Vida, un Destino, Medellín, 1969, pp. 43-47. 
información de un año para maestros graduados delegados por su departamento, permitiéndoles perfeccionarse. Además, por razones financieras y falta de personal docente idóneo, se había centralizado la enseñanza normal en Bogotá, Medellín y Tunja. Esas medidas, generalmente copiadas de modelos europeos, eran bastante inadaptadas a las condiciones del magisterio colombiano y a la situación nacional que exigía una solución rápida, particularmente en el campo.

A fines de 1934 se promulgó la ley 12 creando las escuelas normales rurales. En ellas se daba a niñas con estudios completos de primaria una formación pedagógica de dos años abarcando conocimientos literarios, actividades del campo y desarrollo de la personalidad. En 1938 funcionaban cuatro normales, rurales, en Bogotá, Gigante (Huila), Santa Marta y Popayán con un total de 330 alumnas. A pesar de que muchas de las maestras graduadas en esas escuelas no volvieron al campo, fue una medida que permitió la formación corta del personal docente. En el mismo orden de ideas se crearon en las tres normales nacionales y en varios departamentos cursos de vacaciones para maestros graduados o sin grado, en los cuales recibían una orientación pedagógica hacia los métodos modernos. El Ministerio de Educación decidió también la creación de un servicio de maestros ambulantes, cuyo programa era muy ambicioso. En 1936 debían salir los cien primeros maestros del servicio no para alfabetizar o enseñar, sino para explicar la economía, el comercio, la geografía colombiana a un campesinado en su gran mayoría analfabeta. La segunda parte del proyecto preveía la enseñanza por radio ${ }^{35}$. Este ambicioso programa no se realizó, como tampoco se había logrado implantar el servicio voluntario femenino.

La preocupación del gobierno de López por formar a corto y largo plazo un nuevo magisterio puede apreciarse en la progresión de las partidas nacionales para la enseñanza normalista, que se multiplicaron por más de cuatro entre 1934 y 1938 (ver cuadro No. 9).

Sin embargo, a pesar de que casi todas las resoluciones tomadas por el gobierno de López iban en el sentido de la formación rápida de un magisterio de emergencia, la progresión del número de maestros en esos años $\left(15.2 \%{ }^{36}\right)$ fue bastante menor a la que conocieron los años 1918 a 1934 (promedio anual de 6.25\%). Pero es evidente que los cambios legislativos ocurridos en el período de López no podían tener un efecto inmediato en las estadísticas; es preciso también notar que los requisitos para el nombramiento de nuevos maestros se hicieron entonces más altos.

\footnotetext{
${ }^{35}$ El Tiempo, 22 de noviembre de 1935. La Memoria de 1938 (cuadro en anexo) señala sin embargo, 14 maestros y 11 maestras ambulantes este año.

${ }^{36}$ Esa progresión fue concentrada en el campo. Véase MEN., Memoria de 1938, op. cit., cuadro en anexo.
} 


\section{CUADRO No. 9 \\ PARTIDAS NACIONALES PARA LA ENSEÑANZA NORMAL, 1934-1938. \\ (En pesos)}

$\begin{array}{lcccc}\text { Año } & \begin{array}{c}\text { Escuelas } \\ \text { normales e } \\ \text { instituto } \\ \text { pedagógico }\end{array} & \begin{array}{c}\text { Facultades de } \\ \text { educación o } \\ \text { normal } \\ \text { superior }\end{array} & \begin{array}{c}\text { Cursos de } \\ \text { información }\end{array} & \text { Total } \\ \text { para } & & \\ \text { maestros } & \\ \mathbf{1 9 3 4} & 263.034 & 78.064 & & 341.098 \\ \mathbf{1 9 3 5} & 520.555 & 88.742 & & 609.297 \\ \mathbf{1 9 3 6} & 1.028 .263 & 95.000 & & 1.123 .263 \\ \mathbf{1 9 3 7} & 1.037 .197 & 95.852 & 72.229 & 1.205 .278 \\ \mathbf{1 9 3 8} & 1.256 .322 & 115.000 & 50.000 & 1.421 .322\end{array}$

Fuente: $\quad$ MEN, Memoria de 1938, op. cit., cuadros en anexo.

\section{La Educación Media}

\section{A. El Bachillerato}

A pesar de que la educación secundaria oficial era según la Ley 39 de 1903 costeada, dirigida e inspeccionada por la Nación, una segunda enseñanza estatal no existió en Colombia antes de la década del treinta. Los que se llamaban colegios nacionales eran en realidad colegios dirigidos por comunidades religiosas contratadas por el Estado. El decreto 1951 de 1927 dio fin a esta situación, entregando la totalidad de la secundaria al sector privado y estableciendo la libertad de enseñanza en los límites impuestos por la Constitución. El gobierno seguía sin embargo, financiando becas para los alumnos de escasos recursos económicos. El argumento era que la instrucción pública carecía de dineros y que muchos planteles privados eran mejor administrados que los oficiales. A eso se agregaba la polémica sobre el papel docente del Estado. Respecto a esto, los gobiernos conservadores adoptaron la posición del Vaticano sobre las relaciones Estado Iglesia en educación: el Estado tenía concurrencia supletoria con la Iglesia en la enseñanza primaria, técnica y universitaria, y se abstenía en la secundaria. En resumen, el Estado se limitaba a rellenar los vacíos existentes en la educación privada católica ${ }^{37}$.

Es evidente que los liberales, una vez en el poder, hicieron lo posible para realizar una política opuesta, o sea promover la enseñanza secundaria estatal. Pero las primeras medidas que tomaron solo iban en el sentido de establecer la inspección de la secundaria prevista por la ley orgánica de 1903. A este efecto, ya el decreto No. 1487 de 1932 imponía un examen oficial de ingreso a la universidad, para tratar de nivelar los títulos expedidos por los numerosos colegios privados.

El gobierno de López dio un paso más en el control del Ministerio de Educación sobre la secundaria y decretó que para el reconocimiento oficial de los títulos expedidos por colegios, estos debían adoptar el plan y los programas de estudio prescritos por el Ministerio, tener el material científico y pedagógico mínimo para realizar dichos programas, respetar normas de higiene y educación física, y someterse a la inspección oficial $^{38}$. Ese decreto naturalmente provocó una reacción violenta de la Iglesia, pero el

\footnotetext{
${ }^{37}$ Sobre esa polémica, véase en particular Juan Vicente Huertas y Manuel Juan Huertas, Nuestra Labor y’ Opiniones Respecto a la Educación Nacional, Bogotá, 1930.

${ }^{38}$ Decreto 2214 de 1935 en Educación Colombiana, op. cit., pp. 124-125 
Ministerio, con excepción de algunas concesiones a la Curia, se mantuvo firme en el establecimiento de la inspección secundaria (en 1938 sólo el $20 \%$ de los colegios no eran sometidos a la inspección oficial) y en la obligatoriedad de lose planes oficiales y de los exámenes de ingreso a la Universidad ${ }^{39}$.

Un segundo tipo de medidas fue promover la intervención directa del Estado, estableciendo colegios nacionales. Se crearon dos liceos nacionales, uno para hombres en Zipaquirá, otro para mujeres — creando así el bachillerato femenino— en Chía. Debían servir de modelos para la Nación; pero rápidamente los responsables del Ministerio reconocieron que estos planteles no funcionaban bien y resultaban muy costosos: 264.000 pesos de 1936 a 1939 solamente para su dotación y sostenimiento ${ }^{40}$. La experiencia sobre este proyecto, que debía comprender la creación de varios liceos nacionales para hombres, fue abandonado. La Ley 91 de 1938 creó un nuevo tipo de intervención estatal, a través de la nacionalización de colegios. Los establecimientos que aspiraban a la nacionalización debían funcionar en un edificio adecuado, tener un buen resultado de labores y, en el caso de colegios particulares, tener un mínimo de treinta años de funcionamiento y ser apoyados en su solicitud por el cabildo municipal y el gobernador del departamento. La ley fue un éxito: a fines de 1938 ya había cinco planteles nacionalizados, y cuatro años después siete colegios más.

La principal forma de participación de la Nación en la secundaria siguió siendo, sin embargo, la atribución de becas y auxilios para los colegios, en la cual se nota una progresión entre 1934 y 1938 (ver cuadro No. 10).

En consecuencia, la estructura de la educación secundaria no se modificó mucho en el período. De los 578 establecimientos existentes en 1938, el 83\% eran privados (de los cuales $56 \%$ pertenecían a comunidades religiosas), $5 \%$ municipales, $10 \%$ departamentales y sólo $1 \%$ nacionales. El $31 \%$ de los alumnos concurrían a colegios oficiales, el 69\% a planteles privados. Entre 1934 y 1938 la progresión de la matrícula en la secundaria fue un poco inferior a la de la primaria: $7 \%$ en los colegios oficiales, $9 \%$ en los particulares ${ }^{41}$. En la enseñanza pública, una cuarta parte del alumnado era femenina, mientras que en la privada las mujeres representaban el $48 \%$ del alumnado. Esas cifras nos parecen representar dos tendencias: la primera que los padres de familia de la burguesía preferían orientar a sus hijas hacia colegios privados, por lo general de monjas, preparando futuras amas de casa, que hacia los planteles oficiales que habían iniciado la formación profesional de la mujer, abriéndole el bachillerato $\mathrm{y}$, por consiguiente, la universidad; la segunda, que a pesar de la apertura de la enseñanza secundaria a la mujer que hizo el liberalismo, éste siguió dando prioridad a la formación post-primaria masculina sobre la femenina.

\footnotetext{
${ }^{39}$ Sobre esa polémica, véase más adelante.

${ }^{40}$ MEN., Memoria de 1 938-1939, op. cit.. p. 59.

${ }^{41}$ MEN., Memoria de 1938, op. cit., cuadros en anexo y Luis A. Bermúdez, op. cit., pp. 66-68.
} 


\section{CUADRO No. 10 \\ GASTOS DE LA NACION EN SOSTENIMIENTO, AUXILIOS Y BECAS EN LOS COLEGIOS SECUNDARIOS, 1934-1938. (En pesos).}

Año

\begin{tabular}{rr}
\multicolumn{2}{c}{ Sostenimiento } \\
Colegios \\
1934 & 27.670 \\
1935 & 37.210 \\
1936 & 124.945 \\
1937 & 98.779 \\
1938 & 186.000
\end{tabular}

\section{Auxilios a colegios Becas Departamentales}

61.800

92.901

95.014

103.661

101.964

115.867

Fuente: MEN, Memoria de 1938, op. cit., cuadro en anexo y Memorias de 1938-1939, op. cit., p.81.

\section{B. Otros sectores de Educación Media}

Las enseñanzas agrícola, comercial, industrial y técnica no fueron so-metidas a una reforma profunda y por consiguiente no fueron objeto de polémica alguna durante la administración de López, a pesar de la importancia que él les había acordado en su discurso inaugural. En la legislación hubo pocas modificaciones: en 1937 la enseñanza comercial fue reorganizada y dividida en grado elemental y grado superior equivalente al bachillerato; en 1938 fue fundada la sección industrial en el Ministerio de Educación; además se debatió ampliamente el proyecto de la carrera administrativa, pero fue Eduardo Santos quien firmó la ley de su creación.

Esos tipos de educación medía se enfrentaban a varios problemas que impedían una reforma rápida. En primer lugar, la enseñanza media no clásica estaba en los años treinta en una época de transición entre su forma tradicional y una forma más adecuada a las nuevas condiciones del país. Tradicionalmente, la comercial, femenina, era privada, mal controlada y de mala calidad; la industrial, a cargo de comunidades religiosas, era considerada como beneficencia para las clases pobres y solo ofrecía formaciones preindustriales; la agrícola también carecía de técnica, con excepción de algunas realizaciones semi-oficiales como la Federación Nacional de Cafeteros. En segundo lugar, la creación de las escuelas complementarias en 1932 contribuía a la confusión del Ministerio de Educación en cuanto a la política a seguir. Sin embargo, se puede apreciar una importante progresión cuantitativa en esos sectores de enseñanza media entre 1934 y 1938, aunque la falta de información sobre las características del crecimiento impiden una interpretación cualitativa:

CUADRO No. 11

ENSEÑANZA AGRICOLA, INDUSTRIAL (ARTES Y OFICIOS) Y COMERCIAL, 1935-1939

\begin{tabular}{cccccccccc} 
& \multicolumn{3}{c}{ AGRICOLA } & \multicolumn{4}{c}{ INDUSTRIAL } \\
& \multicolumn{3}{c}{ Oficial } & \multicolumn{2}{c}{ Privada } & \multicolumn{3}{c}{ Oficial } & \multicolumn{2}{c}{ Privada } \\
Año & Esc. & Alumno & Esc. & Alum. & Esc. & Alum. & Esc. & Alum \\
$\mathbf{1 9 3 5}$ & 21 & 88 & 14 & 168 & 4 & 822 & 4 & 415 \\
$\mathbf{1 9 3 6}$ & 15 & 379 & 2 & 73 & 17 & 1.783 & 14 & 748 \\
$\mathbf{1 9 3 7}$ & 7 & 219 & 2 & 207 & 19 & 2.384 & 18 & 1.307 \\
$\mathbf{1 9 3 8}$ & 24 & 777 & 2 & 208 & 24 & 2.685 & 13 & 1.443 \\
$\mathbf{1 9 3 9}$ & 19 & 646 & 3 & 335 & 24 & 2.458 & 13 & 1.055
\end{tabular}

\begin{tabular}{cccc}
\multicolumn{5}{c}{ COMERCIAL } \\
\multicolumn{2}{c}{ Oficial } & \multicolumn{2}{c}{ Privada } \\
Esc. & Alum & Esc. & Alum \\
9 & & 59 & \\
& & & \\
16 & 737 & 94 & 7.460
\end{tabular}


Fuente: Luis A. Bermúdez, op. cit., pp. 70-7 1, MEN, Memoria de 1935, op. cit., p. 254 e Iván Lebot, op. cit., pp. 183 y 185

\section{Las relaciones del Estado y la Iglesia en la Educación}

La política educativa y los intentos reformistas del gobierno de López chocaron fuertemente con varios sectores: las alas moderada y derecha del partido liberal, los conservadores y la Iglesia católica; sin embargo, con esta última el conflicto fue más agudo. La Constitución y el Concordato con la Santa Sede, establecidos por los conservadores, garantizaban enormes prerrogativas a la Iglesia sobre la instrucción pública, mientras dejaban a la educación católica privada sin control estatal. En 1930, después de varias décadas de hegemonía conservadora, cuando el liberal Olaya Herrera fue elegido presidente de la República, su administración de "concentración nacional", no tocó la cuestión de las relaciones entre Iglesia y Estado en educación: sintomáticamente los cinco ministros de educación del período 1930-1934 fueron todos conservadores.

Con Alfonso López Pumarejo, la situación cambió. El partido liberal, en su Convención nacional de 1935, en la cual predominaba el ala reformista del movimiento, se había definido como partido arreligioso y esperaba lograr la separación del Estado y la Iglesia mediante la revisión del Concordato; en materia educativa "el partido liberal considera el programa de la educación nacional como hecho económico y social, y en consecuencia, lucha por el establecimiento de la escuela del trabajo, gratuita, única y obligatoria, teniendo como base la selección del mérito ${ }^{42}$.

El programa de López sería menos ambicioso. Intentaba limitar la vigilancia de la Iglesia sobre la educación pública y establecer un control estatal sobre la instrucción oficial y privada. La mayor realización en este aspecto fue la reforma de la Constitución en 1936. La nueva Carta suprimió la referencia a la religión católica como la oficial de Colombia y garantizó la libertad de cultos. El artículo 14, además de hacer la educación obligatoria, confirmé la libertad de enseñanza, borro toda referencia al control eclesiástico sobre la educación pública, pero estableció la suprema inspección y vigilancia del Estado sobre "los institutos docentes, públicos y privados, en orden a procurar el cumplimiento de los fines sociales de la cultura y la mejor formación intelectual, moral y física de los educandos" ${ }^{43}$. Por otra parte, como lo hemos visto, el decreto No. 1283 y la Resolución No. 179 de 1935 sobre planes de estudio y programas escolares fijaron el contenido mínimo de enseñanza que debían aplicar los planteles públicos y privados para el reconocimiento oficial de sus títulos.

La reacción de la Iglesia no se hizo esperar. El decreto No. 1283 originó una polémica virulenta entre el arzobispo de Bogotá, Monseñor Ismael Perdomo y el entonces ministro de educación, Darío Echandía. El arzobispo denunció el control del Estado sobre el contenido y los métodos de enseñanza como una amenaza contra la libertad de esa y un paso hacia el socialismo. Se opuso al carácter laico y naturalista de los programas, que no acordaban suficiente importancia a la enseñanza de la región y contenían educación sexual. Además, condenó dentro de los programas de literatura y filosofía el recurso a libros censurados por el Vaticano donde se suponía una tendencia materialista. En seguida, el arzobispo exigió que se reconociera a la religión su lugar de preferencia en la orientación de la educación, se renunciara al monopolio estatal sobre esa y se suprimiera

\footnotetext{
42 "Declaración de Principios del Partido Liberal, aprobada por la Comisión Liberal Nacional de 1935" en Dirección Liberal Nacional, Programas y Estatutos del Partido Liberal Colombiano, Bogotá, 1944, p. 29.

43 "Acto Legislativo Reformativo de la Constitución" (5. VIII. 36), en Diego Uribe Vargas, Las Constituciones de Colombia, Madrid, 1977, Tomo II, p. 1106, 
la educación sexual de los programas de Higiene. Darío Echandía, en su respuesta, defendió la vigilancia estatal de la educación, como única manera de impedir los abusos de los particulares, pero acordó a la Iglesia el establecimiento de los programas de religión y aceptó suprimir la educación sexual ${ }^{44}$.

A pesar de estas concesiones, los ataques de la Iglesia y del partido conservador contra la política educativa de López llegaron al paroxismo con la reforma constitucional, adoptada gracias a la abstención conservadora en la Cámara de Representantes. En el primer trimestre de 1936, tanto un documento de la Iglesia ante el Congreso como otro del Directorio Conservador ante el gobierno, confirmaron la estrecha vinculación de los conservadores con la Curia ${ }^{45}$. Los documentos denunciaban la supresión de la invocación de Dios en la Constitución como una falta de respeto a las creencias religiosas más profundas del pueblo colombiano y como una prefiguración de la persecución religiosa del gobierno; al mismo tiempo, con base en la vigilancia estatal sobre la educación decretada en la nueva Constitución, prevenían a los cristianos contra el establecimiento de "la escuela laica, la escuela materialista y sin Dios"; finalmente, anunciaban la resistencia de la Iglesia y del partido conservador frente a la aplicación de dicha Constitución ${ }^{46}$. La respuesta de López $^{47}$ no calmó a la oposición que acusó al gobierno de bolchevismo y casi llamó a la guerra civil. Un texto de Gonzalo Restrepo Jaramillo es muy representativo del estado de espíritu del conservatismo —aun moderado— en aquella época:

"Con pretexto de establecer la supremacía del poder civil, se viene destruyendo aquella armonía de las conciencias que permitió en Colombia el imperio espontáneo de la paz. Es en la escuela donde más peligrosamente se ejerce la labor descristianizadora, y la furia sectaria asalta ya las categorías todas de la vida nacional: condena al hambre a los maestros sembradores humildes de ciencia durante largos años, si no se someten a los códigos de laicismo y de propagación liberal, y lleva su furia hasta las cabezas mismas de los príncipes de la Iglesia, amenazados en su cristiana libertad por el orgullo malindroso de los funcionarios públicos. No meditan ni consideran quienes tales vientos siembran en la pavorosa cosecha de tempestades que preparan para el porvenir inmediato"48.

En efecto, la Iglesia contraatacaría con violencia, especialmente mediante su arma tradicional de mayor eficiencia: el púlpito. En todo el país, los curas párrocos denunciaron ante el pueblo la acción descristianizadora del Estado en la educación pública, incitando a los padres de familia a retirar a sus hijos de las escuelas oficiales. En Antioquia, por ejemplo, departamento profundamente católico, la política eclesiástica tuvo éxito. Los informes de dicho departamento reflejan la preocupación de los secretarios de educación por la campaña antigubernamental desarrollada por los presbíteros. Muchos padres de familia retiraron sus hijos de las escuelas, provocando el cierre de varios planteles por falta de asistencia, especialmente en el campo, donde la influencia de la Iglesia era mayor $^{49}$. Por otra parte, la Iglesia inició una política más ofensiva en el campo de la educación privada católica, fundando nuevas escuelas, colegios y la Universidad Pontificia Bolivariana de Medellín. Se dio también un empuje a la Acción Católica

\footnotetext{
${ }^{44}$ Véase MEN, La Iglesia y el Estado en la Educación Pública, Bogotá, 1935.

${ }^{45}$ El Tiempo, 25 marzo 1936.

${ }^{46}$ El Siglo, 27 febrero 1936 y Revista Javeriana, Vol. V, No. 3, marzo 1936, pp. 161-165. Otros artículos fueron también atacados, como el sobre la propiedad privada.

${ }^{47}$ Véase El Tiempo, 25 marzo 1936.

${ }^{48}$ Gonzalo Restrepo Jaramillo, El Pensamiento Conservador: Ensayos Políticos, Medellín, 1936, p. 176.

${ }^{49}$ Antioquia (Departamento), Dirección de Educación, Informe al Gobernador de 1936, 1937, 1938, 1939 y 1942, Medellín, 19361942. 
Colombiana, dotándola de nuevos estatutos y de una misión educativa y sindical en el campesinado y el proletariado ${ }^{50}$.

También, el conflicto entre la Iglesia y el Estado se manifestó en varios acontecimientos ocurridos durante este período. La escuela normal de institutores de Medellín fue agitada por violentas luchas entre 1934 y 1935; después de la expulsión de un estudiante por haber dictado una conferencia sobre la escuela laica, el Instituto fue objeto de una escandalosa campaña anti-religiosa de varios alumnos que provocó la renuncia intempestiva de su capellán ${ }^{51}$. Aun en Medellín, la creación de la Universidad Pontificia Bolivariana y la negación por parte del arzobispo de dicha ciudad a someter el plantel a otra vigilancia diferente del control papal, provocaron una nueva polémica con el Ministro de Educación Darío Echandía ${ }^{52}$. En el departamento del Tolima, la asamblea departamental de mayoría liberal decreté una ley que excluía del escalafón de maestros a "los individuos pertenecientes a comunidades religiosas" ${ }^{53}$. En 1937, la Nación puso fin al acuerdo con los Jesuitas para la dirección de los colegios de San Bartolomé en Bogotá y San Pedro Claver en Bucaramanga ${ }^{54}$. Los periódicos de estos años abundan en esta clase de sucesos.

Finalmente, después de revelaciones sobre las acciones de ciertas comunidades religiosas contra los indígenas ${ }^{55}$, algunos círculos liberales y el Ministerio de Educación cuestionaron la validez y el rendimiento de la labor educativa desarrollada por las misiones católicas en los territorios nacionales, los cuales escapaban totalmente a la inspección estatal. En 1935, Luis López de Mesa llegó a insinuar que "a medida que los indígenas son más desgraciados, las misiones prosperan notablemente", y puso en cuestión el beneficio de la catequización y castellanización realizada por las comunidades a costa de la identidad cultural de las tribus ${ }^{56}$. Sin embargo, a pesar del nuevo interés del Estado por los territorios nacionales entregados a las misiones, la organización de la educación en esta sección del país no cambió.

\section{Conclusiones}

El balance positivo de la acción del primer gobierno de Alfonso López Pumarejo puede apreciarse en dos aspectos principales: las realizaciones a nivel cualitativo y las realizaciones a nivel cuantitativo; o sea los cambios en la naturaleza de la educación y la progresión numérica de varios indicadores educativos.

En cuanto al primer aspecto, desde luego 1934 inauguré un nuevo período en la educación nacional colombiana. Aunque muchas reformas no fueron propias de la Revolución en Marcha, sino una generalización de experiencias ya realizadas a nivel local desde fines de los años veinte. Entre éstas se destacan la iniciación de los programas de nutrición e higiene en las escuelas primarias y de métodos de enseñanza que tenían más en cuenta la psicología infantil, una relativa mejora de las condiciones del magisterio y la creación de las escuelas normales rurales nacionales. Entre las realizaciones originales

\footnotetext{
${ }^{50}$ La Acción Social Colombiana, después Acción Social Católica, era un organismo de la Iglesia dirigido por los jesuitas encargado de la difusión de la ideología social católica en las clases populares.

${ }^{51}$ El Tiempo, 5 septiembre 1934 y El Siglo, 26 agosto 1935.

${ }^{52}$ El Tiempo, 22 septiembre 1936.

${ }^{53}$ El Espectador, 13 julio 1935.

${ }^{54}$ MEN Memoria de 1938-1939, op. cit., pp. 61-62 y Daniel Restrepo, SJ., La Compañía de Jesús en Colombia, 1589-] 940, Bogotá, 1940, pp. 344-349

${ }^{55}$ Vease por ejemplo Víctor Daniel Bonilla, Siervos de Dios y amos de Indios, Bogotá, 1969.

${ }^{56}$ MEN., Memoria de 1935, op. cit., p. 54. Sobre la educación de los indígenas, véase por ejemplo Gregorio Hernández de Alba, "La Educación en la Guajira", en Educación, Año III, No. 26-2 7. septiembre-octubre 1935, pp. 559-563.
} 
del gobierno de López, seguramente la Comisión de Cultura Aldeana fue la más conocida. Sin embargo, la apertura del bachillerato y de la universidad a la mujer fue más trascendental; el principio de la participación financiera nacional en la construcción de las escuelas primarias inició un cambio importante; finalmente, la influencia de la Iglesia sobre el contenido de los programas educativos fue relativamente limitada y la inspección nacional en la enseñanza secundaria oficial y privada se hizo más efectiva.

En los progresos cuantitativos se inscribe en primer lugar el considerable aumento de los presupuestos nacionales para la educación pública, y entre ellos los destinados al material de enseñanza. También varios sectores de la educación oficial registraron una progresión: la primaria rural, la enseñanza media agrícola e industrial, la normalista nacional y en menor medida la secundaria clásica. Por su parte, la proporción femenina en la educación en general, también registré algún aumento.

Sin embargo, estos logros se hicieron a costa de la educación primaria urbana, la cual se estancó durante el cuatrienio sin poder siquiera seguir la progresión demográfica de la población escolar, y de la enseñanza complementaria que registró una seria regresión. En lo referente al nivel cualitativo, es notable el descuido de la alfabetización, a pesar de que López veía en el analfabetismo del pueblo uno de los principales factores de desintegración social. Muchas reformas que aparecen en la categoría de las realizaciones cualitativas fueron parciales: el alcance de los nuevos programas y métodos de enseñanza fue extremadamente restringido y de ninguna manera se puede decir que el gobierno de López cambió el contenido tradicional de la educación; la inspección nacional de la educación no logró cubrir el país y apartarse de la política; no se consiguió el establecimiento de nuevas relaciones entre el Estado y la Iglesia, sólo posible con una renegociación del Concordato con la Santa Sede.

López tuvo que enfrentarse a la oposición de la Iglesia, del partido conservador y de las filas moderadas y derechas de su propio partido. A nivel de la legislación, fue solo de 1935 a 1937 que López pudo contar con la aprobación de la Cámara de Representantes a los proyectos de su gobierno. Evidentemente dentro de estas dos fechas fue favorecido por la abstención conservadora, mientras que en 1937 el desenvolvimiento de la oposición en el seno de su propio partido le impidió continuar sus propuestas legislativas. A nivel de aplicación de éstas, la resistencia fue siempre fuerte. Esos factores explican muchos de los límites de las reformas educativas y el hecho de que no se consiguió la centralización de la educación nacional propuesta por Luis López de Mesa, la cual hubiera dado mayor radio de acción al Ministerio de Educación. Sin embargo, las dificultades políticas de López no justifican todo. No explican, por ejemplo, cómo la alfabetización no figuró entre las preocupaciones del Gobierno, cómo la educación primaria urbana se estancó, cómo la campaña de nutrición e higiene escolares solo se desarrolló en las zonas urbanas y cómo una parte importante de la nueva legislación permaneció "letra muerta".

La principal explicación nos parece ser la falta de plan educativo del gobierno, reconocida por López:

“¿Debió el Gobierno (...) oír la voz de los técnicos que aconsejaban un esfuerzo metódico, de largos años de actividades encadenadas científicamente, y esperar a que el proceso se cumpliera?"

“¿Debió meditar qué correspondía en la prelación, si hacer escuelas o formar maestros, si normales o universidades, si restaurantes escolares o cultura aldeana? 
Contra esos consejos iniciamos sin pérdida de tiempo todas las reformas, creaciones y campañas que los limitadísimos recursos de que disponíamos nos permitieron acometer" ${ }^{\prime 5}$.

Una multitud de reformas surgieron en todos los campos, sin coordinación ninguna, a veces contradiciéndose entre ellas o careciendo de medios de aplicación. Ocho Ministros de Educación se sucedieron en el cargo, cada uno con las reformas que debían llevar su nombre a la posteridad, de los cuales sólo Luis López de Mesa y Darío Echandía permanecieron en el cargo lo suficiente para dar un mínimo de orientación a la política lopista.

Además, las fuentes de inspiración política de López y de sus aliados eran muy diversas: iban desde el New Deal hasta la Revolución rusa, pasando por el Aprismo peruano, la Revolución mejicana y la República española. A nivel propiamente educativo, los modelos eran importados tanto de Méjico y de la España Republicana como de Bélgica, Italia y Francia, sin adaptación alguna a las condiciones específicas de Colombia.

Otra explicación reside en el electoralismo de ciertas reformas. A pesar de que López declaró que "no va, pues, el liberalismo a ganar elecciones con escuelas" ${ }^{\text {, }}$, el conflicto entre liberales y conservadores por el control de la educación no era desprovisto de consideraciones de clientelismo político: la educación siempre ha sido a largo plazo un medio de formar los electores de mañana y a más corto plazo de conservar el poder. Algunas medidas del gobierno de López, especialmente las dirigidas hacia el campo tales como la creación de la Comisión de Cultura Aldeana, los proyectos de maestros ambulantes y del servicio escolar voluntario, la ley fijando el presupuesto nacional para educación en un mínimo del 10\% del presupuesto general, fueron más declaraciones políticas de principios que expresiones de un cambio estructural del sistema educativo colombiano, pues se abandonaron rápidamente o nunca se cumplieron.

Sin embargo, compartamos con Alfonso López la conclusión sobre la acción educativa de su gobierno:

"No dejaremos millares de escuelas, no terminaremos todas las normales, ni nos tocará inaugurar la Ciudad Universitaria, ni daremos de comer a la totalidad de la población escolar, ni curaremos a los niños palúdicos y sifilíticos en las colonias de vacaciones; pero hemos alcanzado ya el objetivo de crear un afán general en favor de la educación del pueblo"59.

Este comentario visto a la luz de los discursos de 1934 que proclamaban la integración de los sectores marginales a la Nación gracias a la educación, muestra cómo López, ya en 1937, era consciente de los límites de la Revolución en Marcha.

\footnotetext{
${ }^{57}$ Alfonso López P, "Mensaje presidencial al Congreso de 1937" en Mensajes del Presidente López al Congreso Nacional, $1934-$ 1938, Bogotá, 1939, p. 242.

${ }^{58}$ Alfonso López P., 'Mensaje presidencial al Congreso de 1935”, en Ibid., p. 79.

${ }^{59}$ Alfonso López P., Mensaje presidencial al Congreso de 1937”, en Ibid., p. 243. 